\title{
Ambiguità e aggiramento dei problemi come fattori contribuenti all'errore medico
}

\author{
Giulio Marcon 1, Emanuela Barbiero ${ }^{2}$ \\ Risk Manager Clinico. Professore di Management Sanitario e Management Clinico. Università di Padova \\ Risk Manager Clinico. Professore di Infermieristica in area Specialistica. Università di Padova e Università di Udine
}

\begin{abstract}
Administration of the wrong medication is a serious and understudied problem. This article analyzes two cases. The first one describes a patient with iatrogenic hypoglycemia due to administration of the wrong medication: insulin instead of heparin was used to flush the patient's arterial line. In the second one a woman, who was admitted to a teaching hospital for cerebral angiography, mistakenly underwent an invasive cardiac electrophysiology study. The articles analyses strategies to improve patients safety focusing on how removing ambiguity and workarounds can be useful in preventing medication errors.
\end{abstract}

Keywords: medication error, ambiguity, workarounds

How ambiguity and workarounds can contribute to medication error Pratica Medica \& Aspetti Legali 2008; 2(4): 181-184

\section{CASO 1}

La signora G, di 68 anni e non diabetica, venne sottoposta a un intervento chirurgico per bypass aorto-coronarico. Nel corso dell'intervento insorsero alcune complicanze, quali polmonite acquisita, infarto all'emisfero destro, sanguinamento intestinale clinicamente significativo e necrosi tubulare acuta che richiese un intervento di emodialisi. A causa di queste complicanze la paziente fu trattenuta nell'unità di terapia intensiva, dove le sue condizioni si stabilizzarono riportandola in uno stato di benessere. Nel corso della notte seguente l'infermiera di turno, per "pulire" la linea arteriosa occlusa della paziente, utilizzò 1-2 ml di eparina. Tale azione non fu riferita all'infermiera che le subentrò nel turno la mattina seguente.

Nel corso della mattinata la paziente iniziò a muovere il capo e le estremità in modo incontrollato; pertanto l'infermiera di turno, avendo constatato che la sua pressione era $>200 \mathrm{mmHg}$, decise di somministrarle labetalolo. Lo staff della terapia intensiva, accorso quasi immediatamente, diagnosticò una crisi epilettica generalizzata e somministrò lorazepam ev, seguito da midazolam ev e intubò la donna. Fu eseguita la misurazione degli elettroliti sierici e la paziente fu sottoposta a TC per verificare la presenza di emorragia intracranica.

Due settimane dopo l'intervento chirurgico la signora $\mathrm{G}$ fu trovata in stato comatoso e le fu rilevata un'elevata ipoglicemia. Dall'analisi delle azioni occorse nelle ore precedenti, risultò che, a causa della somiglianza dei flaconi, l'infermiera del turno notturno aveva somministrato alla paziente insulina e non eparina [1].

\section{CASO 2}

Una donna di 67 anni fu ricoverata in ospedale poiché doveva sottoporsi a un'angiografia cerebrale. Il giorno successivo al ricovero, l'angiografia fu correttamente eseguita, consentendo di embolizzare uno dei due aneurismi presenti. Il secondo aneurisma richiedeva invece un altro inter- 


\begin{tabular}{|c|c|c|}
\hline Caratteristiche & $\begin{array}{l}\text { Organizzazioni } \\
\text { vulnerabili }\end{array}$ & $\begin{array}{l}\text { Organizzazioni } \\
\text { ad alta performance }\end{array}$ \\
\hline $\begin{array}{l}\text { Progettazione ed } \\
\text { esecuzione del lavoro }\end{array}$ & $\begin{array}{l}\text { Ciò che deve accadere non è chiaro finché } \\
\text { non accade }\end{array}$ & $\begin{array}{l}\text { Le modalità del lavoro sono } \\
\text { attentamente specificate }\end{array}$ \\
\hline Risposta ai problemi & $\begin{array}{l}\text { Le persone fanno quello che è necessario per } \\
\text { completare il lavoro. Non è necessario altro }\end{array}$ & $\begin{array}{l}\text { I problemi sono indagati } \\
\text { immediatamente appena segnalati }\end{array}$ \\
\hline \multirow[t]{4}{*}{ Conseguenze } & \multirow{2}{*}{$\begin{array}{l}\text { Gli effetti dei problemi si propagano in } \\
\text { tutti i settori, e i problemi si ripresentano } \\
\text { regolarmente }\end{array}$} & $\begin{array}{l}\text { Gli effetti dei problemi sono } \\
\text { controllati }\end{array}$ \\
\hline & & \multirow{3}{*}{$\begin{array}{l}\text { Le cause dei problemi vengono } \\
\text { eliminate im modo che i problemi } \\
\text { non si ripresentino }\end{array}$} \\
\hline & $\begin{array}{l}\text { Gli effetti dei processi migliorano } \\
\text { sporadicamente }\end{array}$ & \\
\hline & Le catastrofi accadono periodicamente & \\
\hline
\end{tabular}

Tabella I. Confronto tra le organizzazioni vulnerabili agli errori e le organizzazioni ad alta performance

vento chirurgico che fu quindi pianificato. Dopo l'angiografia, la paziente fu trasferita nel reparto oncologico dove, a seguito di un'identificazione erronea, fu sottoposta a uno studio elettrofisiologico invasivo. Durante l'esecuzione del test i Sanitari si accorsero che si trattava della paziente sbagliata; la procedura fu quindi interrotta e la paziente fu ricondotta nella sua stanza in condizioni stabili [2].

\section{DISCUSSIONE}

Ci si può chiedere se errori medici come quelli dei casi descritti abbiano cause simili. È possibile prendere esempio dalle organizzazioni non sanitarie che cercano di aumentare l'affidabilità delle procedure in modo da superare i problemi della complessità del lavoro, della differenziazione delle conoscenze e della varietà e della volatilità delle circostanze?

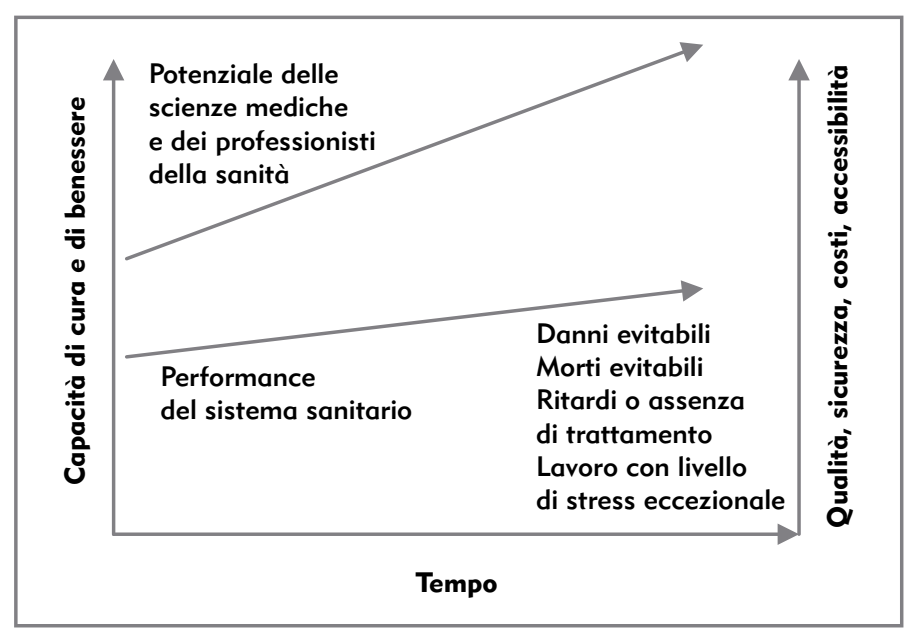

Figura 1. La differenza tra il potenziale e la performance [3]
La risposta a queste due domande è semplice. Le organizzazioni vulnerabili agli errori tollerano l'ambiguità, ossia mancano di fare chiarezza su cosa deve avvenire quando il lavoro procede. Nelle organizzazioni vulnerabili nessuno sa definire esattamente che cosa costituisce un problema, a causa della scarsa conoscenza dei vari aspetti del lavoro. In queste organizzazioni non viene assolutamente chiarito:

- che cosa si propone di raggiungere il gruppo di lavoro;

- chi è responsabile di che cosa;

- come scambiare le informazioni, i materiali o i servizi;

- chi esegue veramente attività, procedure e processi.

In queste organizzazioni, inoltre, quando si manifesta un problema, l'atteggiamento medio è quello di aggirare il problema stesso, cercando ad esempio di mettere in atto qualche soluzione abborracciata o improvvisata, oppure ancora facendo procedere comunque il lavoro anche se gli indicatori dimostrano che la strategia adottata è palesemente sbagliata. Questi accorgimenti, che non sono utili nel ridurre i problemi o nel migliorare i processi, causano solo un'ulteriore confusione.

All'opposto, le organizzazioni vincenti progettano il lavoro come una serie continua di esperimenti, specificando accuratamente le modalità con le quali condurre il lavoro.

La specificazione definisce che cosa deve essere fatto, come deve essere fatto, da chi deve essere fatto, quando deve essere fatto, quali sono i risultati previsti, e permette a tutti i lavoratori di essere sempre connessi con il lavoro e in collaborazione tra di loro.

Quando le specificazioni mancano o si allontanano dall'esperienza corrente, le organizzazioni devono monitorare le deviazioni dei processi per prevenire errori e danni nell'immediato e nel futuro. 


\section{COME L'AMBIGUITÀ ED EVITARE I PROBLEMI CONTRIBUISCONO ALL'INSORGENZA DEGLI ERRORI MEDICI}

La paziente protagonista dell'episodio citato all'inizio dell'articolo si era stabilizzata dopo aver subìto un intervento cardiochirurgico, e si poteva ragionevolmente pensare di pianificare la sua dimissione. Ma l'infermiera che era direttamente responsabile di un processo di cura (il "lavaggio" della linea arteriosa) commette un errore.

La persona che aveva la responsabilità di una parte dei processi di cura della signora (ossia l'infermiera) era responsabile anche del "lavaggio" della linea arteriosa che si stava occludendo. Mentre era chiaro come e con che cosa si doveva "lavare" la linea arteriosa, l'infermiera si era contemporaneamente trovata a dover rispondere ad un allarme che indicava l'occlusione della linea arteriosa di un altro paziente. Purtroppo le fiale dell'insulina e quelle dell'eparina sono molto difficili da distinguere al di fuori della loro confezione, in quanto si tratta di soluzioni incolori e molto simili tra di loro. Linfermiera quindi non aveva alcuna possibilità di sapere se aveva fatto un lavoro corretto. Lo svantaggio dell'aggiramento dei problemi e la mancanza di comunicazione tra professionisti può creare facilmente, come in questo caso, le condizioni per una tragedia.

\section{SISTEMI AD ALTA PERFORMANCE: SPECIFICAZIONE E PROBLEM SOLVING IMMEDIATO}

Nella continua ricerca per migliorare la qualità, la sicurezza e la flessibilità, i leader delle organizzazioni non sanitarie devono specificare esattamente in che cosa consistono i quattro aspetti del lavoro, che sono:

- che cosa si propone di raggiungere il gruppo di lavoro;

- chi è responsabile di che cosa;

- come scambiare le informazioni, i materiali o i servizi;

- chi esegue veramente attività, procedure e processi.

In questo modo, i leader si creano l'opportunità di avere delle sorprese. In primo luogo, permettendo ai lavoratori di riconoscere da soli le deviazioni dalle regole precedentemente specificate, permettono ai lavoratori stessi di tentare nuove modalità di eseguire i lavori stessi. Poi, una volta passata la sorpresa, i leader trattano le discrepanze dalla norma come un qualche cosa che non è normale $\mathrm{e}$ che deve essere immediatamente analizzato e risolto. Questo approccio, apparentemente lassista,

\begin{tabular}{|c|c|c|}
\hline $\begin{array}{l}\text { Stima } \\
\text { di eventi } \\
\text { annui }\end{array}$ & $\begin{array}{l}\text { Benefici se gli } \\
\text { errori fossero } \\
\text { ridotti del } \mathbf{5 0 \%}\end{array}$ & $\begin{array}{l}\text { Benefici se gli } \\
\text { errori fossero } \\
\text { ridotti del } 90 \%\end{array}$ \\
\hline $\begin{array}{c}185.000 \text { pazienti } \\
\text { che subiscono } \\
\text { un danno }\end{array}$ & $\begin{array}{l}92.500 \text { pazienti } \\
\text { che eviterebbero } \\
\text { il danno }\end{array}$ & $\begin{array}{c}166.500 \text { pazienti } \\
\text { che eviterebbero } \\
\text { il danno }\end{array}$ \\
\hline 7.000 morti & 3.500 vite salvate & 6.300 vite salvate \\
\hline
\end{tabular}

Tabella II. Potenziali benefici della riduzione degli errori in ambito sanitario [3]

limita i problemi, genera conoscenza e porta a miglioramenti del lavoro.

Nel caso dell'esame elettrofisiologico che nessuno aveva prescritto, sono stati identificati ben 17 errori, tra cui la mancanza di comunicazione tra le infermiere che seguivano la signora, la comunicazione errata che la signora era stata trasferita e l'omissione da parte dell'ambulatorio di elettrofisiologia di verificare l'identificazione del paziente.

Il pericolo dell'aggiramento dei problemi è evidente proprio nel caso dell'esame elettrofisiologico. In questo caso il pericolo consisteva nella mancanza di richiesta di informazioni, nella mancanza di una prescrizione scritta e documentata, nelle proteste della signora, nella mancanza del consenso informato. Il medico in formazione che seguiva la signora non era nemmeno intervenuto quando era venuto a sapere che il laboratorio di elettrofisiologia aveva iniziato una procedura non richiesta.

Tornando alle organizzazioni non sanitarie, possiamo prendere ad esempio le portaerei, che costituiscono una situazione molto pericolosa a causa delle condizioni atmosferiche, della visibilità limitata, degli improvvisi cambiamenti della missione, dei continui atterraggi e decolli degli aeroplani. Tutte queste attività si svolgono in uno spazio relativamente piccolo costituito dall'area di decollo e atterraggio, in cui sono presenti vari materiali oltre che l'equipaggio. Nonostante tutte queste difficoltà, le operazioni di volo nelle portaerei sono considerate molto sicure. La ragione della sicurezza risiede nel fatto che le attività sono altamente formalizzate anche nel caso in cui un cambiamento di situazione richieda anche un cambiamento dei ruoli. Le uniformi di colori diversi, gli spazi demarcati sul ponte di atterraggio e decollo, la definizione chiara di quali attività devono essere eseguite, prevedono chiaramente che cosa deve essere fatto. Le aberrazioni anche minime, come la posizione errata di qualcuno, rendono chiaro che le operazioni non possono continuare in modo "normale" e devono essere momentaneamente fermate.

La Southwest Airlines è la linea più veloce ed accurata nel processo critico dei decolli, grazie al coordinamento di impiegati specializzati a valu- 
tare molti elementi, come le condizioni meteorologiche, la congestione degli aeroporti, i possibili guasti meccanici, le differenze dei carichi.

Il processo critico specifica che cosa deve essere fatto per garantire un decollo confortevole, e che cosa fare nel caso che si evidenzi un cambiamento della situazione (che costringe ad adottare un piano differente ma già preventivamente formalizzato).

\section{ESEMPI NELLE CURE MEDICHE}

Alcune organizzazioni sanitarie hanno usato con successo la specificazione spinta dei processi. L'Unità di Terapia Intensiva di Salt Lake City ha sviluppato protocolli per migliorare il controllo della glicemia, ridurre le infezioni ospedaliere e ridurre i costi. Questi protocolli vengono regolarmente rivalutati e vengono modificati nel caso in cui si manifestino problemi con il loro uso. È stato quindi dimostrato che è possibile ridurre le ambiguità e l'aggiramento dei problemi. Ad esempio si è osservato in un ospedale che, ad ogni turno, le chiavi dell'armadietto degli stupefacenti venivano cercate in media 23 volte, il che comportava 45 minuti di interruzione del lavoro e il ritardo di somministrazione degli analgesici ai pazienti.

Per evitare le continue ricerche, gli amministratori dell'ospedale hanno allora distribuito chiavi numerate all'inizio di ciascun turno, con regole per evitare la perdita o l'uso errato delle chiavi. Questa procedura ha eliminato le affannose ricerche delle chiavi degli stupefacenti e ha fatto risparmiare 2.895 ore di lavoro degli infermieri in un anno in un ospedale di 350 letti.

\section{EVITARE LE AMBIGUITÀ ED EVITARE DI AGGIRARE I PROBLEMI}

Nel caso della confusione tra eparina e insulina, quali di queste procedure avrebbero permesso di evitare l'errore? Una semplice investigazione avrebbe messo in luce che era facile sbagliare fiala e che era sufficiente separare tra di loro le confezioni di eparina e di insulina per evitare errori gravi.

Se esiste una specificazione precisa di "chi” deve fornire "che cosa" (come materiali, informazioni, servizi), a chi la deve fornire, in quale modo la deve fornire, quando la deve fornire, i problemi possono essere identificati prima che causino eventi avversi. In questo modo le conseguenze dei problemi non si propagano nell'organizzazione e l'investigazione di eventuali errori permette di progettare misure adatte a ridurre il rischio del verificarsi di eventi avversi.

Ma come cominciare, dato che le cure mediche sono di una complessità straordinaria? Ogni paziente presenta caratteristiche proprie, le metodologie diagnostiche e terapeutiche cambiano continuamente, le conseguenze degli errori possono essere gravi, ed ogni paziente richiede cure adeguate alla sua situazione.

È utile cominciare su piccola scala, senza cercare di modificare un intero sistema tutto in una volta. È utile anche cominciare in modo semplice. Tutti i pazienti hanno il diritto di essere curati delle loro malattie o dei loro danni.

Tutti i pazienti hanno il diritto di avere i loro farmaci nel momento giusto, per la via giusta, nella dose giusta. Tutti i pazienti che devono essere sottoposti alle procedure necessarie per guarire delle loro malattie devono essere messi al corrente dei risultati dei loro esami o procedure.

Gli ospedali non devono essere fabbriche o somigliare a delle fabbriche. Gli ospedali devono divenire organizzazioni affidabili, devono essere in grado di formalizzare le attività di cura e devono mettere in atto approcci che permettono di definire e correggere i problemi grazie ad un addestramento continuo nel campo del miglioramento dei processi e del miglioramento delle prestazioni.

Il confronto tra le tra cure mediche e le organizzazioni non sanitarie non ha senso; ciò che ha senso invece è il confronto tra come sono le cure mediche di adesso e come potrebbero essere le cure mediche nel futuro.

\section{BIBLIOGRAFIA}

1. Bates DW. Unexpected hypoglycemia in a critically ill patient. Ann Intern Med 2002; 137: E110-7

2. Chassin MR, Becher EC. The wrong patient. Ann Intern Med 2002; 136: 826-33

3. Spear SS. Fixing healthcare from the inside, today. Cambridge: Institute for Healthcare Improvement, 2005

CORRESPONDING AUTHOR

Prof. Giulio Marcon, e-mail: giulio.marcon@infinito.it 